\title{
BIRD'S EYE VIEW - A 3-D SITUATIONAL AWARENESS TOOL FOR THE SPACE STATION
}

\author{
Adam Dershowitz, Ph.D. and Gregory Chamitoff, Ph.D.
}

United Space Alliance and NASA, Johnson Space Center

\begin{abstract}
Even as space-qualified computer hardware lags well behind the latest home computers, the possibility of using high-fidelity interactive 3-D graphics for displaying important onboard information has finally arrived, and is being used onboard the International Space Station (ISS). With the quantity and complexity of space-flight telemetry, 3-D displays can greatly enhance the ability of users, both onboard and on the ground, to interpret data quickly and accurately. This is particularly true for data related to vehicle attitude, position, configuration, and relation to other objects on the ground or in-orbit. Bird's Eye View (BEV) is a 3-D real-time application that provides a high degree of Situational Awareness for the crew. Its purpose is to instantly convey important motion-related parameters to the crew and mission controllers by presenting 3-D simulated camera views of the International Space Station (ISS) in its actual environment. Driven by actual telemetry, and running onboard, as well as on the ground, the user can visualize the Space Station relative to the Earth, Sun, stars, various reference frames, and selected targets, such as ground-sites or communication satellites. Since the actual ISS configuration (geometry) is also modeled accurately, everything from the alignment of the solar panels to the expected view from a selected window can be visualized accurately.
\end{abstract}

A virtual representation of the Space Station in real time has many useful applications. By selecting different cameras, the crew or mission control can monitor the station's orientation in space, position over the Earth, transition from day to night, direction to the Sun, the view from a particular window, or the motion of the robotic arm. By viewing the vehicle attitude and solar panel orientations relative to the Sun, the power status of the ISS can be easily visualized and understood. Similarly, the thermal impacts of vehicle attitude can be analyzed and visually confirmed. Communication opportunities can be displayed, and line-of-sight blockage due to interference by the vehicle structure (or the Earth) can be seen easily. Additional features in BEV display targets on the ground and in-orbit, including cities, communication sites, landmarks, satellites, and special sites of scientific interest for Earth observation and photography. Any target can be selected and tracked. This gives the user a continual line-of-sight to the target of current interest, and real-time knowledge about its visibility. Similarly, the vehicle ground-track, and an option to show "visibility circles" around displayed ground sites, provide continuous insight regarding current and future visibility to any target.

BEV was designed with inputs from many disciplines in the flight control and operations community both at NASA and from the International Partners. As such, BEV is setting the standards for interactive 3-D graphics for spacecraft applications. One important contribution of BEV is a generic graphical interface for camera control that can be used for any 3-D applications. This interface has become part of the International Display and Graphics Standards for the 16-nation ISS partnership. Many other standards related to camera properties, and the display of 3-D data, also have been defined by BEV. Future enhancements to BEV will include capabilities related to simulating ahead of the current time. This will give the user tools for analyzing off-nominal and future scenarios, as well as for planning future operations. 


\section{Background}

Although the view is reported to be spectacular from onboard the International Space Station (ISS), this view is limited to a few small windows (and the occasional Extra Vehicular Activity). It is thus rather difficult for the crew to determine the relationship of the ISS to the external environment visually. There is numerical data that is provided by the ISS Guidance Navigation and Control (GNC) system to represent the attitude of the station, the location of the station over the earth (State Vector), the direction to the sun, etc. This data is available on the Portable Computer System (PCS) displays, which are text only, but it is not easy to use these individual pieces of information to build good situational awareness. The expression "a picture is worth a thousand words" has basis in the fact that human beings are very quickly able to integrate complex three-dimensional (3-D) information given an appropriate picture.

Bird's Eye View (BEV) is a tool that is able to display such a three dimensional image of the ISS. BEV is run on a Station Support Computer (SSC), and receives data from the station GNC system via a oneway serial cable connection. The SSCs are computers that have limited access to data, and do not run any critical applications. Using the serial connection make it able to display the attitude, location over the earth, direction of the sun, solar array position, etc. BEV started out as a tool for training in robotic operations, and thus had an install base and there was a familiarity with the operating concept. It has since evolved significantly to become MCS BEV (Motion Control System BEV). The version of BEV that is discussed in this paper is the newest version of MCS BEV, and is scheduled to replace the current onboard version soon.

\section{BEV Design Considerations}

The computer hardware available for use onboard the ISS for non-critical applications is limited to Windows 98 on 486-based laptops. These have a rather limited amount of memory and performance. BEV had to be designed to be able to draw 3-D images of the ISS, the earth, the sun, and the stars in realtime, on this platform. In order to do this, the resolution of the 3-D models that are used was reduced significantly from available models.

It was decided that 3-D display tools in use by flight controllers and crew members, in all international partner countries should be consistent in operation, look and feel. In order to accomplish this, meetings were initiated with several different Johnson Space Center (JSC) groups and the other international partners. Together, a set of standards was developed and incorporated into the International Display and Graphics Standards (IDAGS). One of the major outcomes of this was the decision that all control of views (also called cameras) should behave as though the controls act to "fly" or maneuver the virtual camera providing the view, rather than using a "god's-eye-view" perspective, where the controls move the object being viewed. To emphasize this standard the different views in BEV are referred to as different cameras and this pad is called the camera control pad. As many crew members are pilots, this mode of operation is consistent with flying a spacecraft around the outside of the ISS.

\section{Graphical Interface Considerations and Decisions}

The camera control pad that incorporates many of the agreed-upon standards within IDAGS is shown in Figure 1. This pad allows for control of most of the functions of BEV, although the same functions are also available from the keyboard, for quick access to common functions. The corresponding keyboard commands are shown on the cue cards in Figure 2. Note that this pad uses icon menus rather than text menus, across the top, to allow for commonality between the different languages used by the ISS crew. These menus allow for the control of different views, camera modes, objects to be displayed, and targeting. This pad is fairly generic in design and is intended to be used for control of many types of future 3-D applications. 


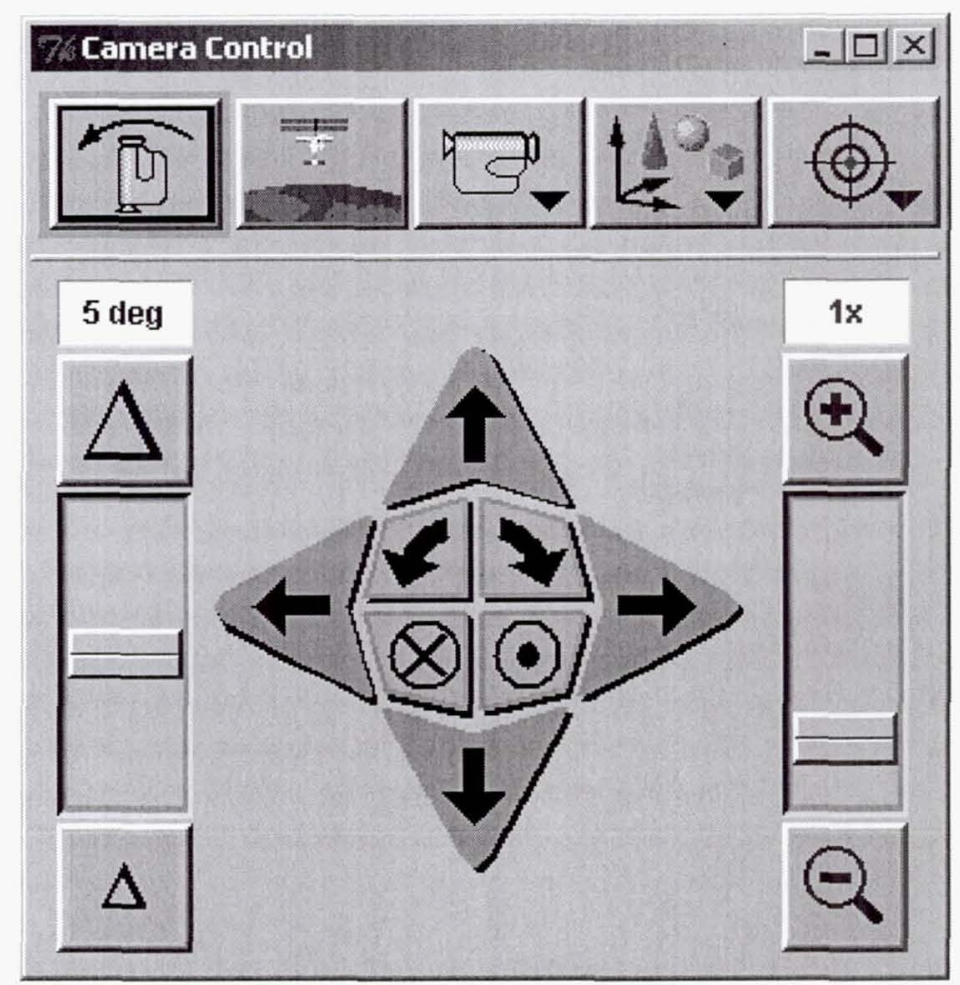

Figure 1 Camera control pad. This pad allows for movement and control of the different views available in BEV.

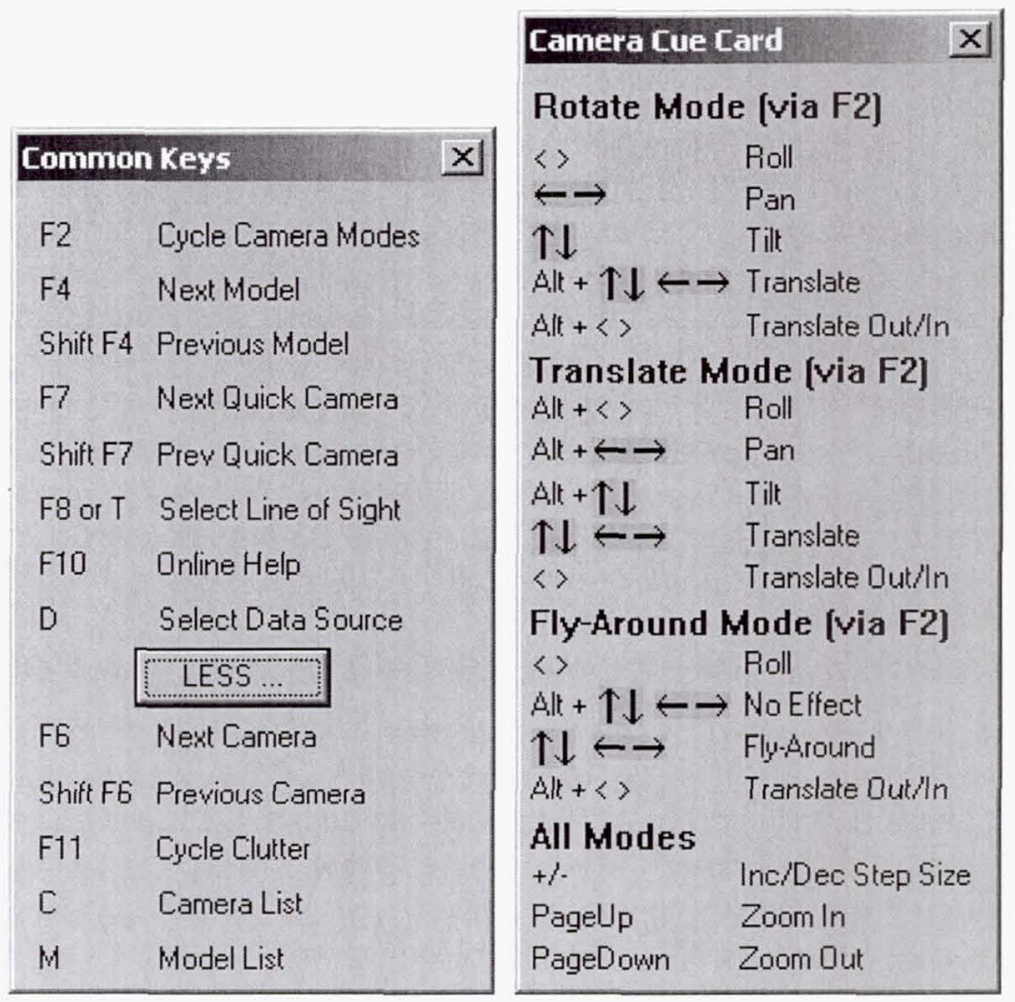

Figure 2 Cue cards for the commands available in BEV.

The main part of the pad is the central cruciform region of buttons that are used to move the camera view. The slider on the left side changes the step size, while the slider on the right zooms the camera view in or 
out. There are three different modes in which BEV cameras can operate. One, the controls can simply translate the camera. Two, the controls can rotate the camera. Three, a "fly-around" mode is available that combines the other two modes and allows the user to keep the camera pointed at the same location on the station, while it moves around. This may be thought of as having the camera fly on the outside surface of an invisible sphere, while pointing to the center of it at all times, after selecting a point on the station as this center point. Cameras may be based on a few types of reference frames, such as the Local Vertical-Local Horizontal (LVLH) frame, or an ISS body frame (i.e. Down).

\section{BEV Requirements and Features}

There are many requirements in order for BEV to be useful in flight, and in the Mission Control Center (MCC). In addition, through use, a large number of additional features have been added to further increase its utility. BEV is primarily a 3-D display tool, so it therefore must have an accurate 3-D ISS model and earth model. These models must contain enough resolution to be visually convincing, yet must be updated every second, with live data, on a limited computer platform. The models must articulate the large elements, such as the solar arrays. There must be models for all the different station configurations that are present during constructions. The direct lighting must be accurate, so BEV includes the sun in the correct location and shadowing due to the earth (i.e. day and night). For pointing use, BEV must be able to display the location of relevant ground sites, and draw a line-of-site vector towards them, as well as toward the sun and toward the TDRS communication satellites. BEV uses actual star locations to allow the crew to use their familiarity with stars used as attitude reference points, and is able to draw a vector toward any named star. As BEV is intended as a motion control related application it must also be able to display some text information about the state of the ISS motion control system, such as the attitude and reference frame in use. An image of a typical BEV window is shown in Figure 3.

Data is provided to BEV in two different ways. Data in the MCC is provided over a network using the Information Sharing Protocol (ISP). This same protocol is used internal to several of the onboard computer systems, such as PCS. Thus, BEV must be able to use ISP data to receive real-time data. BEV is also used as the 3-D display component of several other tools. For this to be the case, it is necessary for $\mathrm{BEV}$ to have an additional binary interface. This interface allows for BEV to be driven from Matlab, or any other application that supports this interface. 


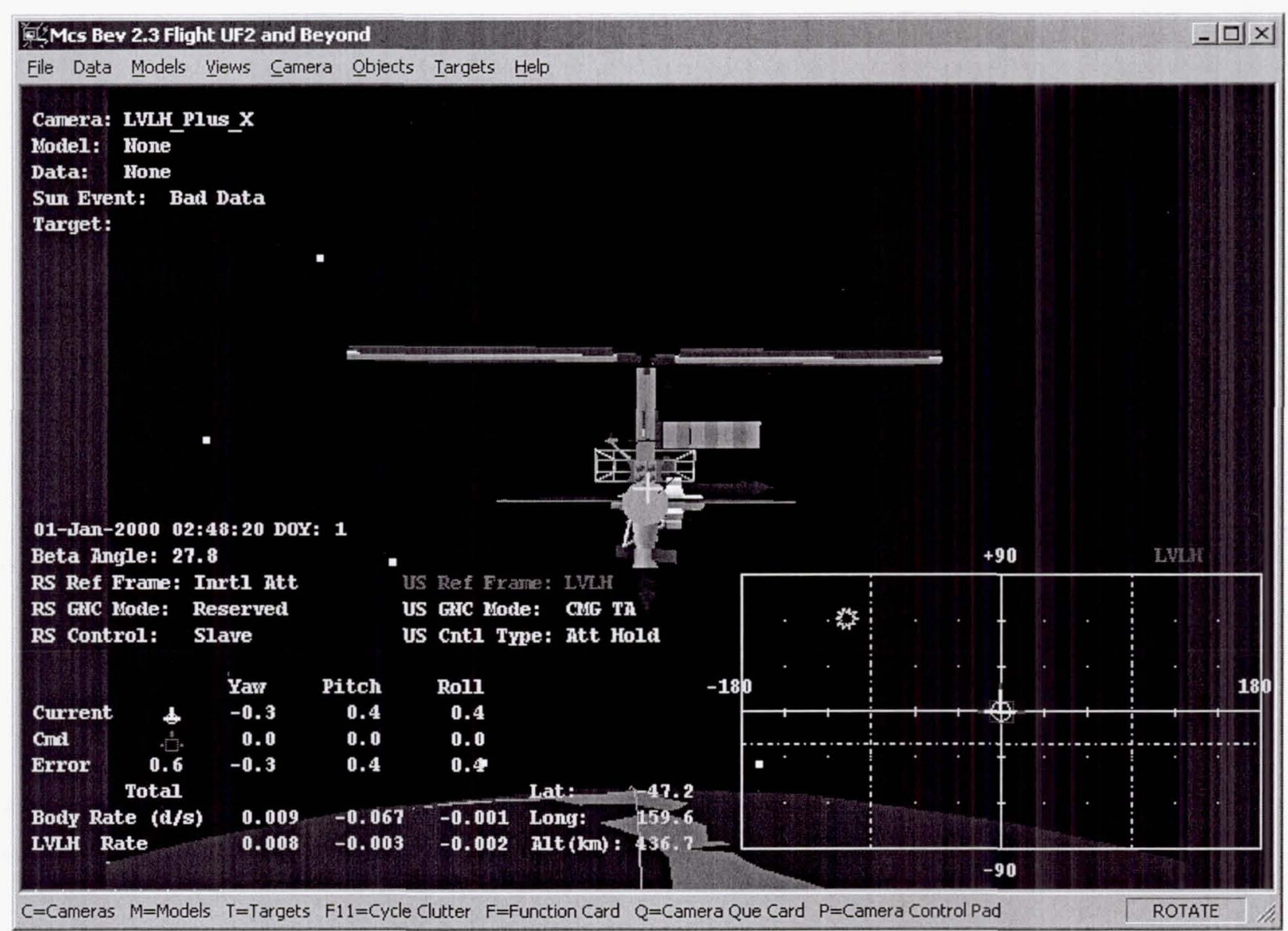

Figure 3 A typical single window BEV display.

BEV is able to display 1, 2, or 3 different views at the same time. An example of 3 views is shown in Figure 4. In this case, the user has turned on the display of a large amount of text information showing such things as the attitude and rates of the ISS. In addition, the display at the lower right shows a graphical depiction of the attitude of the station. Here the azimuth and elevation of the white icon in the rectangular yellow box is used to represent the attitude and the blue icon represents the commanded attitude. It is also able to show the direction towards a selected target and toward the sun. The dotted horizontal line in this display represents the horizon. 


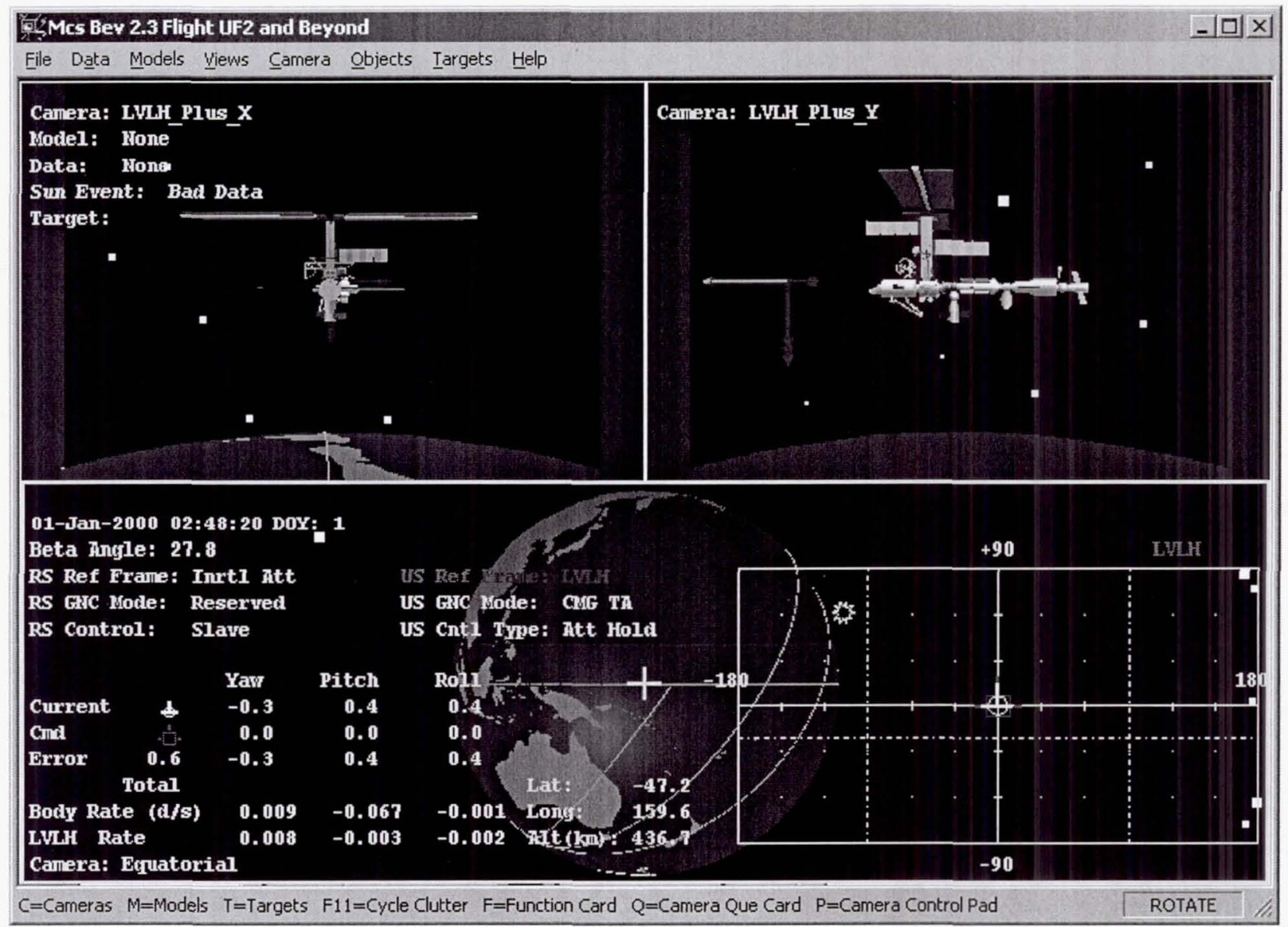

Figure $4 \mathrm{BEV}$ with three views, and corresponding text information displayed.

BEV also includes the ability to propagate a state vector (position, velocity, and time). This is handled in two different ways. The user is able manually to input a state and attitude into the associated program, Bird's Eye view Extended state Determination (BirdSeed), which then propagates this state in real time. The BirdSeed propagator interface is shown in Figure 5. It allows the user to display past or future states. Additionally BEV has a built-in propagator, which was added recently, that automatically takes over to supply state data to BEV when ISP data is not available, such as during communication blockage (LOS) or due to onboard computer problems. This second propagator has no user interface, other than the ability to disable it. When using either of these propagators, the attitude is simply assumed by BEV to remain constant.

BEV has several features in order to aid crew members in photographing ground targets, and to help with awareness of upcoming ground coverage events. BEV is able to display targets in several different categories, (Control Centers, Photo Opportunities, Landmarks, Cities, Communication Sites, stars, etc.) For each of these categories the user has the option to display icons on the earth, and/or visibility range rings. Additionally the user can elect to have a line-of-site vector drawn from the station to a selected individual site, or to the next visible site in one of the categories. Several of these features are shown in Figure 6. In this example, the dashed line from the station represents the direction to the next available target. The dashes mean that this target (indicated in the upper left segment as American Samoa Reefs) is not yet visible. However it will be visible in 5 minutes and 49 seconds as can be seen in text information in the upper left. The yellow arrow visible in both of the upper displays represents the direction towards the sun. The red circles, visible in the lower view, represent the visibility range rings around the targets. Each target will be visible to the ISS when the ISS is contained within the corresponding ring. In addition 
the light blue line, visible in the lower view, represents the ground track for the next three orbits, allowing an easy indication of upcoming targets.

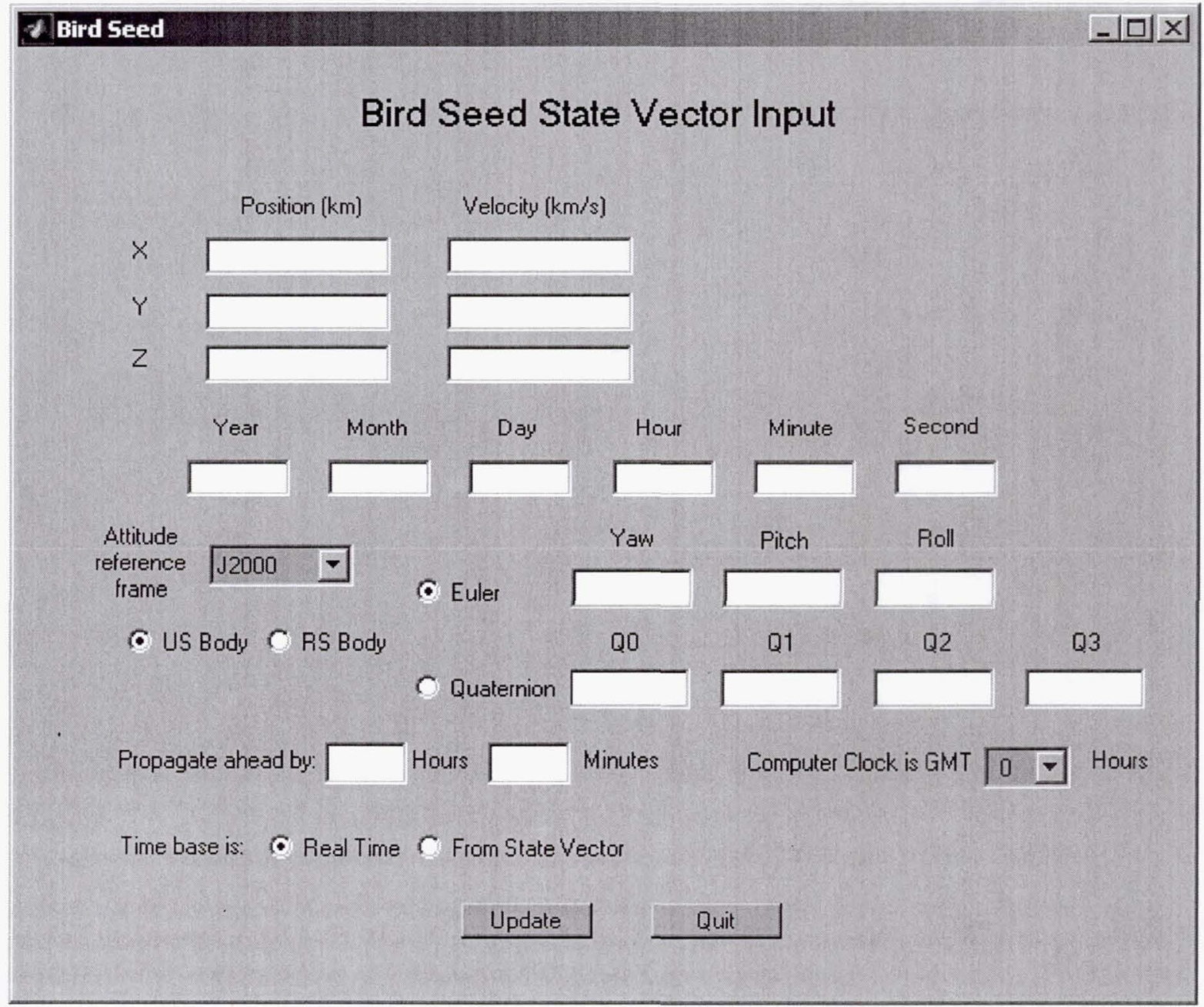

Figure 5 BirdSeed propagator input window. 


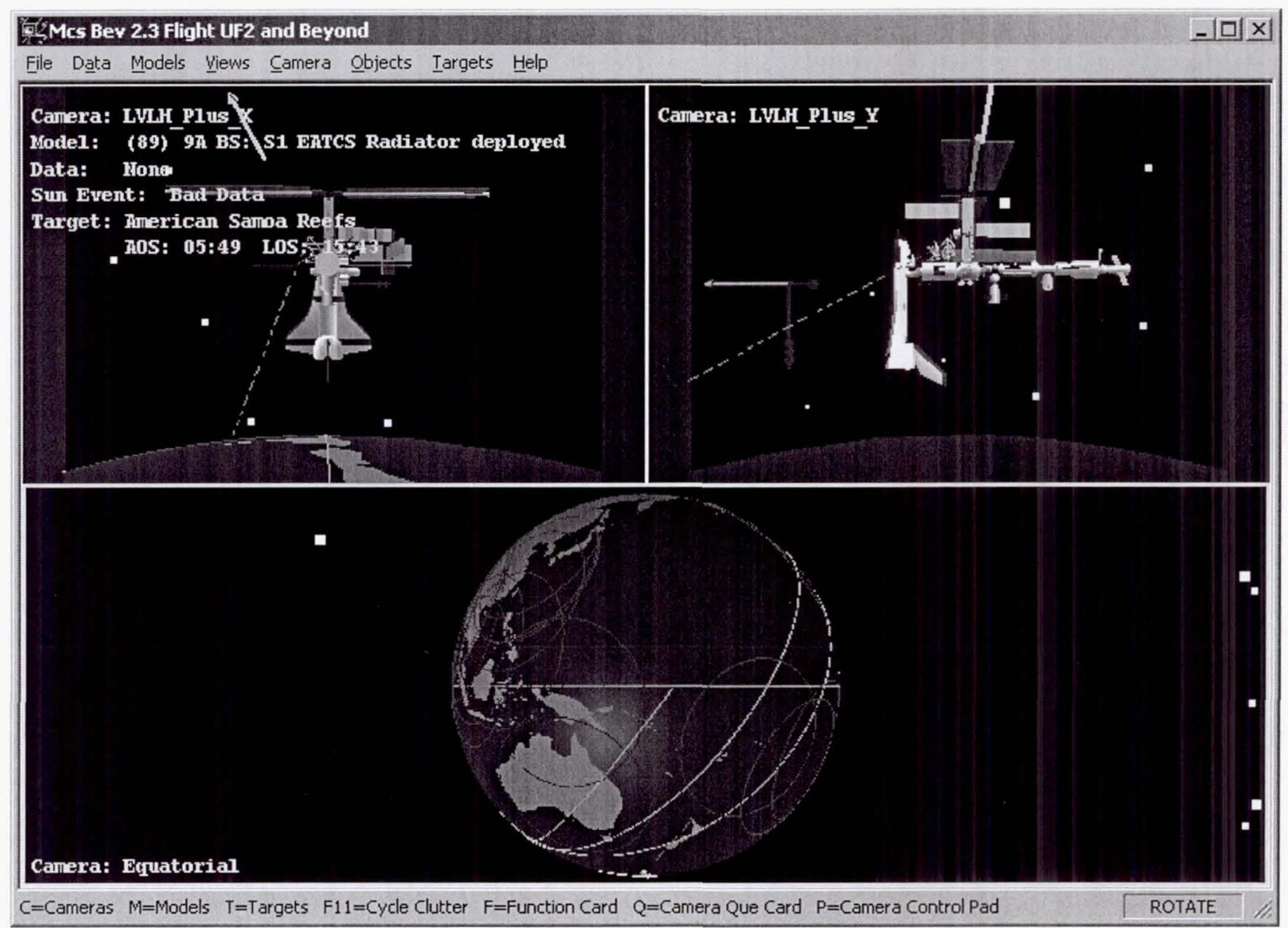

Figure 6 BEV with Line-Of-Site vector shown and visibility circles.

\section{Uses of BEV}

BEV may be used for many different tasks onboard ISS, and in MCC. Primarily, it is able to provide general situational awareness. It is the quickest way to determine the approximate attitude of the ISS. For example, when the ISS was recently in free drift for a significant amount of time after an unsuccessful docking attempt of a Progress the Flight Director on duty requested that the BEV display be projected on the large screen at the front of the MCC. This was done in order to provide both him, and the rest of the flight control team, with an understanding of the attitude of the station and the overall situation that would not have been possible without a 3-D graphical tool.

The first use of BEV onboard ISS was by the first commander, Bill Shepherd. In one case he wanted to understand the attitude of the ISS during an upcoming docking with a Progress resupply vehicle. At that early construction phase of the ISS, data was not available to BEV, as there was not yet a U.S. motion control system. However, "Shep" was able to manually input the predicted state vector and attitude, then to use that to visualize the upcoming docking, the location over the ground, the lighting due to the relative sun location, and the expected direction of the incoming vehicle. This mental image allowed him to be prepared for the upcoming docking.

Appendage pointing and blockage are difficult to predict analytically. For a given attitude BEV allows an easy display of the angle of the solar arrays and the location of an antenna and the direction to a satellite. This makes it clear whether an antenna is being blocked by a solar array, or if a solar array, or other part of the station, is in direct sunlight or not. This allows for thermal concerns to be predicted. The Attitude 
Control, Power, Communication and Thermal groups, in MCC, have all taken advantage of BEV while on console.

The ISS crew is given a list of photo opportunities that they are supposed to take advantage of when possible. In order to take these photos they must determine when the target will be visible, as well as from what window etc. BEV is able to display a circle around any of the ground sites, and then to draw a line-of-site vector towards that site. When the site is not yet visible this line is drawn as a dashed line, which changes to a solid line when the target is visible. Times to visibility and loss-of-visibility are also displayed. This allows for the crew to know when to prepare to take a photo, and what direction to expect the target. Additionally BEV is able to show the view from a selected station window, based on the current station attitude. This allows for a crew member to understand if a given window is pointed in a direction that will allow for the desired sighting.

\section{Current BEV Status and Future Work}

Currently, BEV is available in the MCC, in office at JSC, and onboard the ISS. There are some issues with providing data to BEV that are in the process of being solved. A training class was developed for the ISS crews to learn to use BEV. The first crew to receive that complete lesson is Expedition 5, the current ISS crew. The prior crews received limited training and were not thoroughly familiar with BEV. It is hoped that BEV will become the primary 3-D display and situational awareness tool for use in the MCC. There are several other tools that are being considered, but none of them have the large number of features and current availability for use of BEV.

There are several tasks that are required of flight controllers that a visualization tool would aid in. For example, the selection of an attitude for docking of a Progress to the ISS is a complicated and iterative process among different flight controller disciplines in the U.S. and Russia. BEV allows for proposed docking attitudes to be quickly displayed and analyzed.

Several different tools have been considered that will leverage BEV, by using it as the 3-D graphics component, and using the binary data interface to drive it. In order to allow for different attitudes to be compared, a tool that could quickly switch between them would be ideal. If there were an easy way to just click and drag to select different attitudes, it would be very easy to select an optimum attitude for certain purposes. For example, if a user could simply rotate the station in 3-D by moving a mouse, then it would be easy to find an attitude that keeps a certain component as cold as possible, by keeping it out of the sun. This type of task is currently extremely difficult.

Flight controllers are required to convert between many different reference frames (LVLH, J2000 (inertial), Shuttle, etc.) In addition, much of the attitude data that is used is expressed as quaternions, which are computationally easier to use, but extremely difficult to intuitively understand. There are tools to do these conversions, however the results are difficult to verify, except using the same tools. A tool that displayed the results graphically would allow for an easy visual confirmation of the expected results. This type of "common sense" check is critical for data reliability, but is extremely difficult to do without a tool that allows for an easy understanding.

BEV does not currently have a 2-D map like display, so the crew also uses another tool called World Map. This is one of the features that will be incorporated into a future version of BEV.

\section{Conclusions}

Today, we are very used to live video of "interesting events" and expect to be able to see things for ourselves. It is not possible to actually see the attitude of the space station (except when another vehicle is in close proximity). BEV, however, is a simulation that is able to provide the same critical information about the current ISS situation, in a quick glance. It is expected that BEV will be used as the primary 
display of an SSC computer, similar to a screen saver. In this way, the crew will only have to glance over, from anywhere in the module, to understand their current location over the earth and attitude.

Among crew, flight controllers, and flight directors, there has been consistent positive response to BEV, with questions about how to get it sooner, and suggestions of how it could be made even better. Humans can be trained to interpret very complicated information. However, when that same information can be presented in simple intuitive ways there is less required training and fewer errors. BEV is an application whose strength springs from an intuitive desire to just look at things rather than to interpret numbers and draw a mental image. When presenting information to people, especially critical information, the data should be displayed in an intuitive way. Numerical information is rarely intuitive, especially when it represents 3-D information. Consistent 3-D displays provide much better information and should be used whenever possible. 